\title{
Effect of Random Antenna Element Displacements on Sparse-UCA-Root-MUSIC Direction-of-Arrival Estimation
}

\author{
Veronique Inghelbrecht* \\ veronique.inghelbrecht@ugent.be
}

\author{
Hendrik Rogier ${ }^{+}$ \\ hendrik.rogier@ugent.be
}

\author{
Jo Verhaevert ${ }^{+}$ \\ jo.verhaevert@ugent.be
}

\author{
Marc Moeneclaey*
marc.moeneclaey@ugent.be \\ Marc Moeneclaey*
marc.moeneclaey@ugent.be
}

\author{
Tanja Van Hecke ${ }^{+}$ \\ tanja.vanhecke@ugent.be
}

\author{
Dries Vande Ginste ${ }^{+}$ \\ dries.vandeginste@ugent.be
}

Herwig Bruneel*

hb@telin.ugent.be

\begin{abstract}
We leverage a generalized polynomial chaos (GPoC) expansion to model the effect of correlated random antenna element displacements in a uniform circular antenna array (UCA) on the probability density function of the estimated directions-of-arrival (DOAs). To limit the number of antenna array realizations to be evaluated through full-wave simulations, we determine the GPoC expansion coefficients based on the stochastic collocation method. The method yields a speedup factor of about 40 compared to the Monte-Carlo approach, for a UCA with two displaced antenna elements and for DOA estimates obtained via the sparse-UCA-root-MUSIC algorithm.
\end{abstract}

Index Terms-Antenna arrays, direction-of-arrival estimation, stochastic collocation method (SCM)

\section{INTRODUCTION}

Most algorithms for direction-of-arrival (DOA) estimation using circular antenna arrays (UCAs) exploit the circular symmetry of the array. When random antenna displacements break this symmetry, these algorithms experience a significant performance loss. In this letter, we apply the stochastic collocation method (SCM) to obtain the mean square error (MSE) of the DOA estimates. The procedure is illustrated by relying on the Sparse UCA Root-MUSIC [1] DOA estimation algorithm. This algorithm can compensate for mutual coupling by incorporating all relevant phase modes of the active elements patterns of the antenna arrays.

\section{SpARSE UCA Root-MUSIC Algorithm FOR A UCA With ANTENNA DisplaCEMENTS}

The nominal UCA consists of $N$ identical antenna elements distributed over a circle with radius $R$. The phase center of each antenna element is located in the $x y$-plane, at azimuth angles $\varphi_{n}=(n-1) \frac{2 \pi}{N}$ with $n=1,2, \ldots, N$. The number of incoming plane waves equals $K$, with corresponding elevation and azimuth angles $\left(\theta_{k}, \varphi_{k}\right), k=1, \ldots, K$. In order to account

This research was partially funded by the Inter-University Attraction Poles Program initiated by the Belgian Science Policy Office.

* Dep. of TELIN, Ghent University.

+ Dep. of INTEC, Ghent University. for the antenna element displacements, we adjust the received signals $\mathbf{x}$ as follows [2]:

$$
\mathbf{x}(t)=\mathbf{A}_{\text {displ }} \mathbf{s}(t)+\mathbf{v}(t), t=0,1,2, \ldots
$$

where $\mathbf{s}(t) \in \mathbb{R}^{K \times 1}$ is the transmitted signal vector at time instant $t, \mathbf{x}(t) \in \mathbb{C}^{N \times 1}$ is the received signal vector and $\mathbf{v}(t) \in$ $\mathbb{C}^{N \times 1}$ is assumed to be a spatially uncorrelated additive white Gaussian noise vector. The signal-to-noise ratio SNR (in $\mathrm{dB}$ ) is defined as:

$$
\mathrm{SNR}=10 \log _{10}\left(\frac{E\left\{\|\mathbf{A} \mathbf{s}(t)\|^{2}\right\}}{E\left\{\|\mathbf{v}(t)\|^{2}\right\}}\right),
$$

with $\mathbf{A}$ the element-manifold matrix of the nominal UCA. The $k$-th column of the $(N \times K)$ matrix $\mathbf{A}_{d i s p l}$ equals the element-space manifold vector $\mathbf{a}_{\text {displ }}\left(\theta_{k}, \varphi_{k}\right)$, determined by the DOA $\left(\theta_{k}, \varphi_{k}\right)$ of the $k$-th plane wave. $\mathbf{A}_{d i s p l}$ incorporates both the effects of the displacements of the antenna elements, as well as the mutual coupling effects, and can be found using a electromagnetic field simulator such as 4NEC2. On the received signals (1), we apply the sparse UCA Root-MUSIC algorithm [1], assuming a perfect UCA configuration.

\section{Stochastic Collocation Method}

In order to investigate the influence of Gaussian random displacements, we apply the SCM method [3]. First, we apply a Karhunen-Loève transform [4] to decorrelate the Gaussian random variables: the vector $\mathbf{u}$ of $O$ correlated displacements with covariance matrix $\boldsymbol{\Sigma}$ is related to a vector $\boldsymbol{\eta}$ of $O$ statistically independent variables through $\mathbf{u}=\sqrt{2} \mathbf{V} \sqrt{\Lambda} \eta$, where the columns of $\mathbf{V}$ are the eigenvectors of $\boldsymbol{\Sigma}$, and $\boldsymbol{\Lambda}$ is the diagonal matrix containing the corresponding eigenvalues. Consider a vector $\mathbf{y}(\mathbf{u})$ depending on the displacement vector u. We can rely on the Askey scheme [5] to approximate $\mathbf{y}(\sqrt{2} \mathbf{V} \sqrt{\boldsymbol{\Lambda}} \boldsymbol{\eta})$ as a truncated polynomial expansion of the variables $\boldsymbol{\eta}$ :

$$
\mathbf{y}(\sqrt{2} \mathbf{V} \sqrt{\boldsymbol{\Lambda}} \boldsymbol{\eta}) \approx \sum_{i_{1}, \ldots, i_{O}} \mathbf{y}_{i_{1}, \ldots, i_{O}} H_{i_{1}}\left(\eta_{1}\right) \ldots H_{i_{O}}\left(\eta_{O}\right)
$$


where $H_{i}(v)$ represents the Hermite polynonial of degree $i$, and the summation index $i_{l}$ ranges from 0 to $P_{l}(l=$ $1, \ldots, O)$; hence, the expansion (3) has a degree $P_{t o t}=$ $P_{1}+\ldots+P_{O}$. Considering that the Hermite polynomials satisfy $\int H_{i}(v) H_{j}(v) e^{-v^{2}} d v=\delta_{i, j}$ with $\delta_{i, j}$ representing the Kronecker delta function, it follows that the coefficients $\mathbf{y}_{i_{1}, \ldots, i_{O}}$ in (3) are given by:

$$
\mathbf{y}_{i_{1}, \ldots, i_{O}}=\int \mathbf{y}(\sqrt{2} \mathbf{V} \sqrt{\boldsymbol{\Lambda}} \boldsymbol{\eta}) H_{i_{1}}\left(\eta_{1}\right) \ldots H_{i_{O}}\left(\eta_{O}\right) p(\boldsymbol{\eta}) \mathrm{d} \boldsymbol{\eta} .
$$

We approximate the integral (4) by cubature formulas with weight function $e^{-|\boldsymbol{\eta}|^{2}}$ ( [6]). Note that, if we want to calculate the mean of $\mathbf{y}(\mathbf{u})$, we can evaluate it as $E[\mathbf{y}(\mathbf{u})]=$ $\sum_{k=1}^{Q} w_{k} \mathbf{y}\left(\mathbf{u}_{\mathbf{k}}\right)$, with $\mathbf{u}_{k}$ the $Q$ quadrature points, and $w_{k}$ the corresponding weights ( [6]). Consequently, if the vector $\mathbf{y}(\mathbf{u})=\left[y_{1}(\mathbf{u}), \ldots y_{K}(\mathbf{u})\right]$ represents the MSE of the $K$ DOA estimates:

$$
y_{i}(\mathbf{u})=E_{\boldsymbol{v}}\left[\left(\theta_{i}-\hat{\theta}_{i}\right)^{2} \mid \mathbf{u}\right], i=1, \ldots, K,
$$

for a certain value of the displacement vector $\mathbf{u}$ (averaged over different values of the noise vector $\mathbf{v}$ ), we obtain the MSE averaged over both the displacement vector and the noise vector as:

$$
\mathrm{MSE}=E\left[\left(\theta_{i}-\hat{\theta}_{i}\right)^{2}\right]=\sum_{k=1}^{Q} w_{k} E_{\boldsymbol{v}}\left[\left(\theta_{i}-\hat{\theta}_{i}\right)^{2} \mid \mathbf{u}_{k}\right] .
$$

\section{Numerical Results}

Consider a uniform circular array consisting of nine dipole antenna elements tuned to $2.45 \mathrm{GHz}$ (dipole length $l=\lambda / 2=$ $6.12 \mathrm{~cm}$ ). All antennas are terminated by a load $Z_{0}=73 \Omega$. In the center of the circle, there is a short-circuited dipole with length $l=\lambda / 2$, representing a platform effect. The nine array elements are uniformly distributed on a circle with radius $\lambda / 2$. We consider $K=3$ uncorrelated sources, each transmitting pseudorandom bit sequences of length 500, using BPSK modulation. These signals are impinging on the UCA along the same elevation angle $\theta=90^{\circ}$ and along the azimuth angles $\left[20^{\circ}, 200^{\circ}, 285^{\circ}\right]$. The signals are received in the presence of additive white Gaussian noise $\mathbf{v}(t)$. We consider the case where the first and second antenna element have random radial displacements $u_{1}$ and $u_{2}$, while the other antenna elements remain at their nominal positions. These random variables are assumed to be multivariate Gaussian distributed with zero mean, correlation coefficient $\rho=0.8$, and standard deviation $\sigma$.

In Fig. 1, we have plotted the MSE of the first true $\mathrm{DOA}=20^{\circ}$ as a function of the signal to noise ratio (SNR) and for different values of the dispersion. To obtain the mean square error, we apply two different methods: Monte-Carlo (M-C) simulation and the two-dimensional SCM theory. For the M-C method, based on 10000 realizations of $\left(u_{1}, u_{2}, \mathbf{v}\right)$, we estimate the 3 DOAs and calculate the corresponding MSE. We compare this with the two-dimensional SCM theory that relies on the cubature formulas for the plane with $Q=44$ quadrature points. In each of these quadrature points $\left(u_{1}, u_{2}\right)$

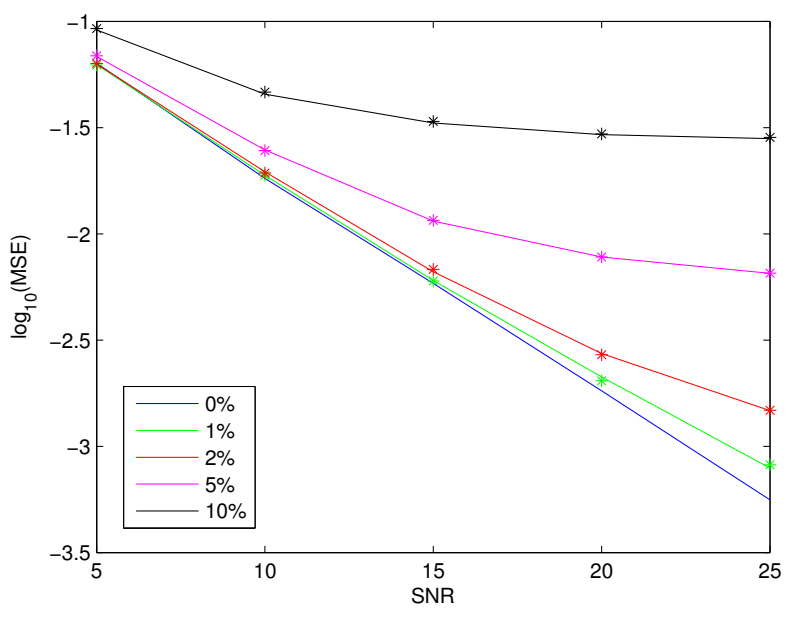

Fig. 1. The $\log _{10}(\mathrm{MSE})$ as a function of SNR, obtained with M-C (solid line) method and SCM (asterisk) for a radial displacement of the first two antenna elements, $\left(u_{1}, u_{2}\right)$ Gaussian distributed with standard deviation $[0 \%, 1 \%, 2 \%, 5 \%, 10 \%]$ of the radius.

we calculate the MSE averaged over 1000 values of the noise vector $\mathbf{v}$ (5), to finally obtain the MSE given by (6). Note that, for the SCM-method, we have to calculate the matrix $\mathbf{A}_{\text {displ }}$ only in the 44 quadrature points, whereas for the $\mathrm{M}-\mathrm{C}$ method, we need to calculate $\mathbf{A}_{\text {displ }}$ for all 10000 realizations. Due to cost of an electromagnetic field simulation, the calculation of the $\mathbf{A}_{\text {displ }}$ is by far more time consuming than applying the Sparse-UCA-Root-MUSIC algorithm. We observe from Fig. 1 that the displacements give rise to a MSE floor, which increases with increasing standard deviation of the displacement. The results from M-C simulation and SCM theory match very well.

\section{CONCLUSIONS}

We have investigated an UCA, subject to correlated Gaussian random displacements. We have applied the SCM method and the M-C method to obtain the $\log _{10}(\mathrm{MSE})$ as a function of the SNR. Comparison of the SCM to the M-C method demonstrates that the SCM method accurately predicts the performance of the DOA estimates, with a reduction in CPU time by a factor of 40 .

\section{REFERENCES}

[1] R. Goossens and H. Rogier, "UCA Root-MUSIC with sparse uniform circular arrays," IEEE Trans. Signal Process., vol. 56, pp. 4095-4099, Aug. 2008.

[2] A. Balanis, Antenna Theory Analysis and Design. Hoboken, New Jersey: A John Wiley \& Sons, 2005.

[3] V. Inghelbrecht, J. Verhaevert, T. Van Hecke, H. Rogier, M. Moeneclaey, and H. Bruneel, "Stochastic framework for evaluating the effect of displaced antenna elements on DOA estimation," IEEE Antennas and Wireless Propagation Letters, vol. 16, pp. 262-265, 2017.

[4] T. El-Moselhy, Field Solver Technologies for Variation-Aware Interconnect Parasitic Extraction, 2005.

[5] D. Xiu, "Fast numerical methods for stochastic computations: A review," Communications in Computational Physics, vol. 5, no. 2-4, pp. 242-272, Feb. 2009.

[6] R. Cools, L. Pluym, and D. Laurie, "Cubpack++: A C++ package for automatic two-dimensional cubature," ACM Transactions on Mathematical Software (TOMS), vol. 23, no. 1, pp. 1-15, Mar. 1997. 